\section{Novel role for COX2 inhibitors in the treatment of N141I PSEN2 familial Alzheimer's disease?}

Evidence from epidemiological studies suggesting a potential protective effect of antiinflammatory drugs in Alzheimer's disease (AD) have not been supported by therapeutic studies using nonsteroidal anti-inflammatory drugs. To further investigate the role of antiinflammatory drugs in familial AD (FAD), a group led by Pasinetti studied the potential association between cyclooxygenase (COX) and AD pathogenesis in neuronal cell lines expressing mutant N141I presenilin-2 (PSEN2). These associations were then studied in the brains of patients harboring the mutation.

N141I PSEN2 expression was found to coincide with a greater than fourfold increase in expression of the inducible form of COX2 but not in the constitutively expressed COX1, and with a subsequent increase in the generation of COX2-derived prostaglandin $\mathrm{E}_{2}$ in neuronal cells. Similar responses were found in the brains of FAD cases harboring N141I PSEN2 mutations. The expression of the N141I PSEN2 FAD gene strongly promoted glycogen synthase kinase-3 $\beta$ activity coincidental with a reduction in the level of $\beta$-catenin translocated from the cytoplasm to the nucleus. Inhibition of COX2-mediated generation of prostaglandin $E_{2}$ in the neuronal cells using nimesulide, a preferential COX2 inhibitor, protected against N141I PSEN2-mediated apoptotic neuronal cell death. This protection was associated with inhibition of glycogen synthase kinase- $3 \beta$ activity and subsequent normalization of $\beta$-catenin distribution.

The study provides support for the potential pharmacogenomic identification of N141I PSEN2 FAD cases that might be most likely to benefit from inhibition of COX2 during the progression of clinical FAD dementia.

Carol Lovegrove

Original article Qin W et al. (2006) Inhibition of cyclooxygenase as potential novel therapeutic strategy in N141I presenilin-2 familial Alzheimer's disease. Mol Psychiatry 11: 172-181

\section{Recognizing early signs of meningococcal disease}

Meningococcal disease progresses rapidly; early diagnosis and treatment is therefore very important. As early symptoms of this disease can be difficult to distinguish from those of common self-limiting infections, however, many children are initially misdiagnosed. To improve understanding of the early signs of this devastating disease, researchers in the UK investigated the pre-hospital sequence of its symptoms in children.

Questionnaires and interviews were used to collect data from parents of 448 children aged $\leq 16$ years who had had meningococcal disease between December 1997 and February 1999 (103 fatal, 345 nonfatal cases).

Median time between onset (first symptoms) and hospital admission was $13 \mathrm{~h}$ in children under 1 year of age, increasing with age to up to $22 \mathrm{~h}$ in those aged $15-16$ years. Fever was the first symptom in children aged $<5$ years; those aged $\geq 5$ years experienced headache first. All age-groups showed early symptoms common to many viral illnesses, such as loss of appetite, irritability, nausea and vomiting; these symptoms generally occurred $4-8 \mathrm{~h}$ from onset. The first specific clinical features of meningococcal disease were signs of sepsis (leg pain, cold hands and feet, abnormal skin colour), which generally occurred within $12 \mathrm{~h}$ of onset. Classic meningitis symptoms (e.g. rash, neck stiffness, photophobia) occurred later, around 12-15h from onset, followed by unconsciousness, delerium and seizures at $16-22 \mathrm{~h}$.

The authors conclude that leg pain, cold hands and feet, and abnormal skin colour are also important clinical signs of meningococcal disease in children $\leq 16$ years. They urge that these early signs are recognized by both parents and primary-care clinicians, as the widely recognized, classic symptoms only occur later in the disease course, thereby delaying vital early hospital treatment.

Rebecca Ireland

Original article Thompson MJ et al. (2006) Clinical recognition of meningococcal disease in children and adolescents. Lancet 367: 397-403

\section{Could blockade of brain-derived neurotrophic factor be used to treat neuropathic pain?}

Tactile allodynia is a type of neuropathic pain that occurs after peripheral nerve injury, and is induced by signals from activated spinal 